(C) 1984. The Genetical Society of Great Britain

\title{
SELF-INCOMPATIBILITY IN RYEGRASS \\ IX. CROSS-COMPATIBILITY AND SEED-SET IN AUTOTETRAPLOID LOLIUM PERENNE L.
}

\author{
C. H. FEARON*, M. D. HAYWARD $\dagger$ AND M. J. LAWRENCE* \\ *Department of Genetics, University of Birmingham, Birmingham B15 2TT. †Welsh Plant \\ Breeding Station, Plas Gogerddan, Aberystwyth SY 23 3EB
}

Received 16.xii.83

\section{SUMMARY}

The results of the experiments presented in the two previous papers of this set showed that 35 autotetraploid ryegrass plants fell into 18 different incompatibility classes and, in particular, that only one $S-Z$ pair in the pollen needs to be matched in the stigma for incompatibility to occur (Fearon, Hayward and Lawrence, $1984 a, b$ ). These results were obtained by pollinating tetraploids with pollen from diploids and vice versa. In the experiment described in the present paper, the classification and conclusions of these earlier experiments are put to the test by crossing the tetraploid plants inter se. The results completely confirm those of the earlier experiments. In addition, they show that there is no dominance between the alleles at either locus in the pollen and that incompatibility only occurs when both an $S$ and a $Z$ allele are matched in the stigma.

Two implications of these results are considered. First, that only one $S-Z$ pair in the pollen needs to be matched in the stigma strongly suggests that the growth of incompatible pollen is positively inhibited, rather than that of compatible pollen being positively stimulated.

Second, it is shown theoretically that a consequence of this mechanism is that the level of cross-compatibility is appreciably lower in populations of tetraploids relative to those of diploids in which few, but equally frequent alleles are segregating at each locus. If the number of alleles that are segregating in the population exceeds twelve, however, this difference in level of cross-compatibility becomes negligible.

The implications of this outcome for plant breeding are briefly considered.

\section{INTRODUCTION}

The results of an analysis of the inheritance and expression of self-incompatibility in two families of autotetraploid ryegrass, given in the two previous papers, showed that 35 plants examined fell into 18 different classes, and that only one $S-Z$ pair in the pollen need be matched by the same combination in the stigma for incompatibility to occur (Fearon, Hayward and Lawrence $1984 a, b)$. These results were obtained by observing the pollination relationships between the tetraploids and two sets of related diploids, first by observing the reaction of pollen from the latter on stigmas of the former and second, by reversing this procedure. Hitherto, we have not examined the pollination relationships among these tetraploids. Since the information obtained from the first two stages of this analysis can be used to predict the compatibility of a cross between any pair of tetraploids, it is clear that the results obtained by inter-pollinating these tetraploids provide an important, confirmatory test of our conclusions. The chief purpose of the present paper is to give the results of an experiment that was designed with this argument in mind. 
A further critical test of these conclusions is to show that the expected relationships between the predicted compatibility of the cross and seed-set holds. Accordingly, the seed-set results obtained by selfing and crossing members of the tetraploid families are also presented.

\section{Materials AND Methods}

Each of the 35 legitimate tetraploids, whose incompatibility genotype was determined in the first experiment, was included in the present one, together with three of the open-pollinated progeny (Fearon et al., 1984a). These plants were crossed in as many combinations as could be made in the time available, the combinations being chosen so as to include each of the expected compatibility levels in the experiment. Some of the pollinations were performed in 1980 and the majority in 1981. Each plant was also selfed. In all, 463 in vitro crosses and selfs were examined. Technical details for this part of the experimental work were the same as those given in previous papers (Fearon et al., loc cit.).

In the spring of 1982, clonal replicates of these plants were crossed for seed, using the techniques given in Cornish, Hayward and Lawrence (1980). Crosses were again chosen so as to include each of the levels of compatibility expected on each hypothesis. In addition, 28 plants were selfed.

\section{THEORY}

The expected level of compatibility of a cross between any pair of tetraploids depends, as in diploids, on the number of alleles at each locus in the male that are not present in the female parent of the cross in question. Fifteen types of cross can be recognised, ranging from a pair where all of the alleles of the male also occur in the female to the case where there is complete non-identity in this respect. Only 12 of these 15 types of cross can be made among the classes of the present experiment.

Two modes of action of the incompatibility alleles in the pollen were considered in the previous paper; the first, hypothesis 1 , was that incompatibility occurs when only one $S-Z$ pair in the pollen is matched in the stigma, whilst hypothesis 2 required the matching of both alleles at each locus. The results of the previous experiment led to the rejection of the latter. The inter-pollination of the tetraploids in the present experiment, however, allows an even better discrimination between the two hypotheses. Accordingly, the expected levels of cross-compatibility on each of these models are shown in table 1 for the twelve types of cross available.

The expected compatibilities shown in table 1 have been derived on the assumption that no male gametes are doubly reduced or are aneuploid and that there is no dominance in the pollen. The present experiment provides a test of the third assumption. The effects of double reduction and of aneuploidy on these expectations are similar to those described in the previous paper, namely, that both, if present, would very slightly increase the level of compatibility expected on hypothesis 1 and very slightly decrease that expected on hypothesis 2 (Fearon et al., 1984b). However, it is again most unlikely that failure of either of these assumptions would have, at most, more than a marginal effect on our ability to discriminate in practice 


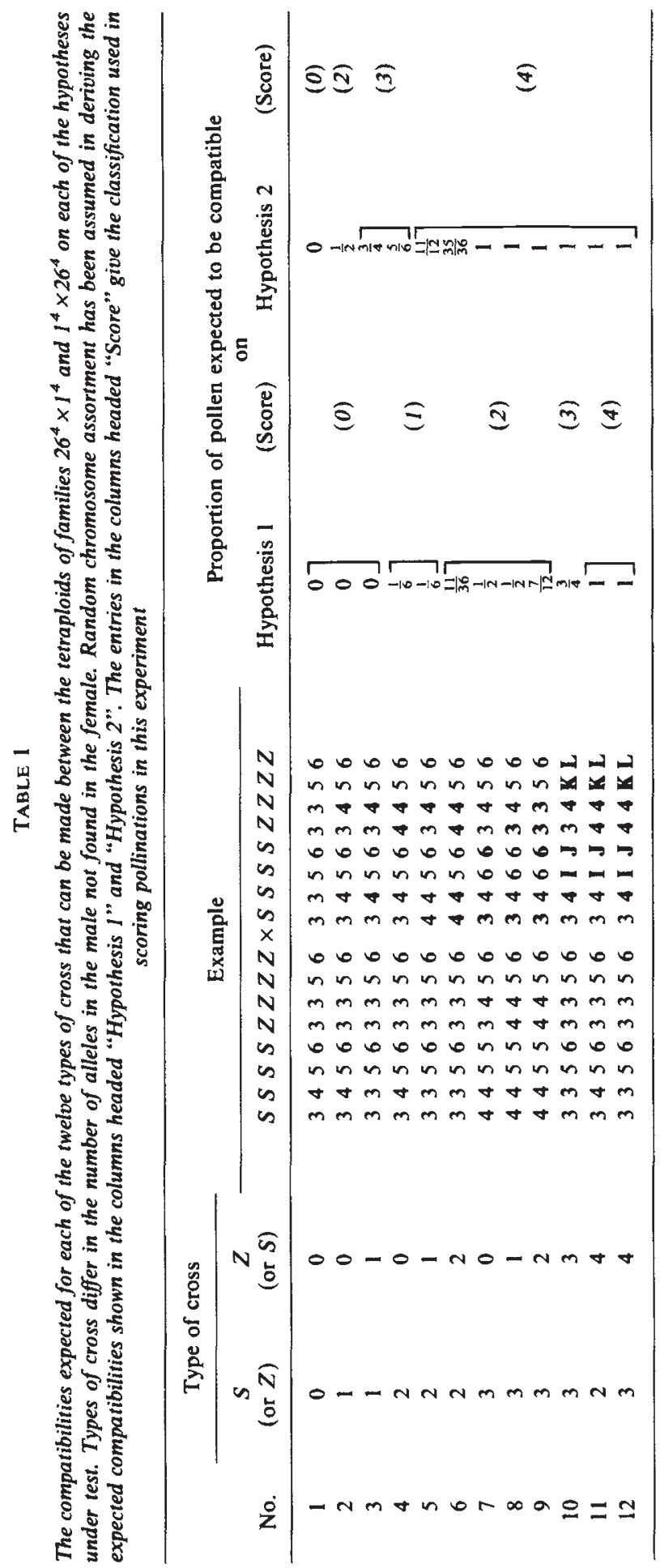


between these hypotheses (see Scarrott, 1981, for a more detailed discussion of this matter).

In practice, it is unlikely that we should be able to distinguish between those pollinations that are expected to be $\frac{11}{36}, \frac{1}{2}$ and $\frac{7}{12}$ compatible on hypothesis 1 ; nor between those that are expected to be $\frac{3}{4}$ and $\frac{5}{6}$ compatible or between those that are expected to be $\frac{11}{12}, \frac{35}{36}$ and fully compatible on hypothesis 2 . Hence we can expect to recognise only five kinds of pollination on the first and four kinds on the second hypothesis; these are shown in parenthesis under the heading "Score" in the last two columns of table 1. This loss of information, however, has only a small effect on our ability to discriminate between the hypotheses, because, whereas many pollinations between plants of different genotype are expected to be incompatible on hypothesis 1 , very few are on hypothesis 2 ; a difference of this magnitude should be easy to detect in practice. The pattern of pollinations expected on each hypothesis when plants of families $26^{4} \times 1^{4}$ and $1^{4} \times 26^{4}$ are crossed are shown in tables 2 and 3.

\section{Results}

\section{(i) Cross-compatibility}

The results obtained by crossing plants of these families (fig. 1) are clearly quite inconsistent with the expectations of hypothesis 2 (table 3 )

TABLE 2

The pattern of pollinations expected on hypothesis 1 when plants of family $26^{4} \times 1^{4}$ and its reciprocal are crossed, with the pollinations shown on the four point scale shown in table 1. (Plants with alleles designated $I, J, K$ and $L$ are the open-pollinated progeny whose full genotype is not known)

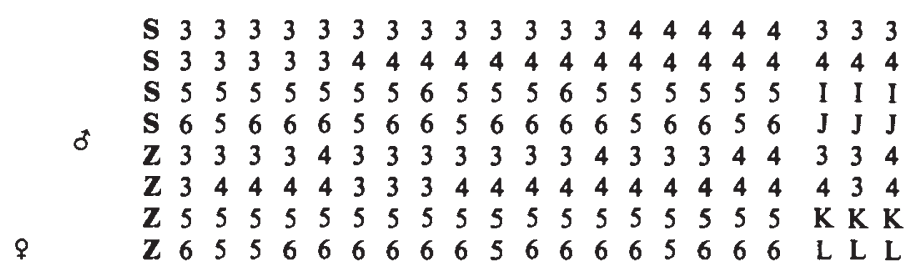

$\mathbf{S} \mathbf{S} \mathbf{S} \mathbf{S} \mathbf{Z} \mathbf{Z} \mathbf{Z} \mathbf{Z}$

\begin{tabular}{llllllllllllllllllllllllll|lll}
3 & 3 & 5 & 6 & 3 & 3 & 5 & 6 & 0 & 0 & 0 & 0 & 1 & 0 & 0 & 0 & 0 & 0 & 0 & 0 & 1 & 1 & 1 & 1 & 2 & 2 & 3 & 2 & 4
\end{tabular}

\begin{tabular}{llllllll|llllllllllllllllll|lll}
3 & 3 & 5 & 5 & 3 & 4 & 5 & 5 & 0 & 0 & 0 & 0 & 0 & 0 & 1 & 2 & 0 & 1 & 1 & 2 & 1 & 1 & 2 & 2 & 1 & 2 & 2 & 2 & 2
\end{tabular}

\begin{tabular}{llllllll|lllllllllllllllllllllllllll}
3 & 3 & 5 & 6 & 3 & 4 & 5 & 5 & 0 & 0 & 0 & 0 & 0 & 0 & 0 & 0 & 0 & 0 & 0 & 0 & 0 & 1 & 1 & 1 & 1 & 1 & 2 & 2 & 2
\end{tabular}

\begin{tabular}{llllllll|llllllllllllllllll|lllll}
3 & 3 & 5 & 6 & 3 & 4 & 5 & 6 & 0 & 0 & 0 & 0 & 0 & 0 & 0 & 0 & 0 & 0 & 0 & 0 & 0 & 1 & 1 & 1 & 1 & 1 & 2 & 2 & 2
\end{tabular}

\begin{tabular}{llllllll|llllllllllllllllll|lll}
3 & 3 & 5 & 6 & 4 & 4 & 5 & 6 & 1 & 0 & 0 & 0 & 0 & 1 & 1 & 1 & 0 & 0 & 0 & 0 & 0 & 1 & 1 & 1 & 1 & 1 & 3 & 4 & 2
\end{tabular}

\begin{tabular}{llllllll|llllllllllllllllll|llll}
3 & 4 & 5 & 5 & 3 & 3 & 5 & 6 & 0 & 0 & 0 & 0 & 1 & 0 & 0 & 1 & 0 & 0 & 0 & 1 & 1 & 0 & 0 & 0 & 1 & 1 & 2 & 2 & 4
\end{tabular}

\begin{tabular}{llllllll|llllllllllllllllll|lll}
3 & 4 & 5 & 6 & 3 & 3 & 5 & 6 & 0 & 0 & 0 & 0 & 1 & 0 & 0 & 0 & 0 & 0 & 0 & 0 & 1 & 0 & 0 & 0 & 1 & 1 & 2 & 2 & 4
\end{tabular}

\begin{tabular}{llllllll|llllllllllllllllll|lll}
3 & 4 & 6 & 6 & 3 & 3 & 5 & 6 & 0 & 1 & 0 & 0 & 1 & 1 & 0 & 0 & 1 & 0 & 0 & 0 & 1 & 1 & 0 & 0 & 2 & 1 & 2 & 2 & 4
\end{tabular}

\begin{tabular}{llllllll|llllllllllllllllll|lll}
3 & 4 & 5 & 5 & 3 & 4 & 5 & 6 & 0 & 0 & 0 & 0 & 0 & 0 & 0 & 1 & 0 & 0 & 0 & 1 & 0 & 0 & 0 & 0 & 0 & 0 & 2 & 2 & 2
\end{tabular}

\begin{tabular}{llllllll|llllllllllllllllll|lll}
3 & 4 & 5 & 6 & 3 & 4 & 5 & 5 & 0 & 0 & 0 & 0 & 0 & 0 & 0 & 0 & 0 & 0 & 0 & 0 & 0 & 0 & 0 & 0 & 0 & 0 & 2 & 2 & 2
\end{tabular}

\begin{tabular}{llllllll|lllllllllllllllllllll}
3 & 4 & 5 & 6 & 3 & 4 & 5 & 6 & 0 & 0 & 0 & 0 & 0 & 0 & 0 & 0 & 0 & 0 & 0 & 0 & 0 & 0 & 0 & 0 & 0 & 0 & 2 & 2 & 2
\end{tabular}

\begin{tabular}{llllllll|llllllllllllllllll|lll}
3 & 4 & 6 & 6 & 3 & 4 & 5 & 6 & 0 & 1 & 0 & 0 & 0 & 1 & 0 & 0 & 1 & 0 & 0 & 0 & 0 & 1 & 0 & 0 & 1 & 0 & 2 & 2 & 2
\end{tabular}

\begin{tabular}{llllllll|llllllllllllllllll|lll}
3 & 4 & 5 & 6 & 4 & 4 & 5 & 6 & 1 & 0 & 0 & 0 & 0 & 1 & 1 & 1 & 0 & 0 & 0 & 0 & 0 & 0 & 0 & 0 & 0 & 0 & 2 & 4 & 2
\end{tabular}

\begin{tabular}{llllllll|llllllllllllllllll|lll}
4 & 4 & 5 & 5 & 3 & 4 & 5 & 6 & 2 & 1 & 2 & 2 & 2 & 0 & 1 & 2 & 0 & 1 & 1 & 2 & 1 & 0 & 0 & 0 & 0 & 0 & 2 & 2 & 2
\end{tabular}

\begin{tabular}{llllllll|llllllllllllllllll|lll}
4 & 4 & 5 & 6 & 3 & 4 & 5 & 5 & 1 & 1 & 1 & 1 & 1 & 0 & 0 & 0 & 0 & 0 & 0 & 0 & 0 & 0 & 0 & 0 & 0 & 0 & 2 & 2 & 2
\end{tabular}

\begin{tabular}{llllllll|llllllllllllllllll|lll}
4 & 4 & 5 & 6 & 3 & 4 & 5 & 6 & 1 & 1 & 1 & 1 & 1 & 0 & 0 & 0 & 0 & 0 & 0 & 0 & 0 & 0 & 0 & 0 & 0 & 0 & 2 & 2 & 2
\end{tabular}

\begin{tabular}{llllllll|llllllllllllllllll|lll}
4 & 4 & 5 & 5 & 4 & 4 & 5 & 6 & 2 & 1 & 2 & 2 & 2 & 1 & 2 & 2 & 0 & 1 & 1 & 2 & 1 & 0 & 0 & 0 & 0 & 0 & 3 & 4 & 2
\end{tabular}

\begin{tabular}{llllllll|llllllllllllllllll|lll}
4 & 4 & 5 & 6 & 4 & 4 & 5 & 6 & 2 & 1 & 1 & 1 & 1 & 1 & 1 & 1 & 0 & 0 & 0 & 0 & 0 & 0 & 0 & 0 & 0 & 0 & 3 & 4 & 2
\end{tabular} 
TABLE 3

The pattern of pollinations expected on hypothesis 2 when plants of family $26^{4} \times 1^{4}$ and its reciprocal are crossed, with the pollinations scored on the four point scale shown in table 1. Other details as table 2

\begin{tabular}{|c|c|c|c|c|c|c|c|c|c|c|c|c|c|c|c|c|c|c|c|c|c|c|c|c|c|c|c|}
\hline & & & ㅇ & ? & $\delta$ & $\begin{array}{l}\mathbf{S} \\
\mathbf{S} \\
\mathbf{S} \\
\mathbf{S} \\
\mathbf{Z} \\
\mathbf{Z} \\
\mathbf{Z} \\
\mathbf{Z}\end{array}$ & $\begin{array}{l}3 \\
3 \\
5 \\
6 \\
3 \\
3 \\
5 \\
6\end{array}$ & $\begin{array}{l}3 \\
3 \\
5 \\
5 \\
3 \\
4 \\
5 \\
5\end{array}$ & $\begin{array}{l}3 \\
3 \\
5 \\
6 \\
3 \\
4 \\
5 \\
5\end{array}$ & $\begin{array}{l}3 \\
3 \\
5 \\
6 \\
3 \\
4 \\
5 \\
6\end{array}$ & $\begin{array}{l}3 \\
3 \\
5 \\
6 \\
4 \\
4 \\
5 \\
6\end{array}$ & $\begin{array}{l}3 \\
4 \\
5 \\
5 \\
3 \\
3 \\
5 \\
6\end{array}$ & $\begin{array}{l}3 \\
4 \\
5 \\
6 \\
3 \\
3 \\
5 \\
6\end{array}$ & $\begin{array}{l}3 \\
4 \\
6 \\
6 \\
3 \\
3 \\
5 \\
6\end{array}$ & $\begin{array}{l}3 \\
4 \\
5 \\
5 \\
3 \\
4 \\
5 \\
6\end{array}$ & $\begin{array}{l}3 \\
4 \\
5 \\
6 \\
3 \\
4 \\
5 \\
5\end{array}$ & $\begin{array}{l}3 \\
4 \\
5 \\
6 \\
3 \\
4 \\
5 \\
6\end{array}$ & $\begin{array}{l}3 \\
4 \\
6 \\
6 \\
3 \\
4 \\
5 \\
6\end{array}$ & $\begin{array}{l}3 \\
4 \\
5 \\
6 \\
4 \\
4 \\
5 \\
6\end{array}$ & $\begin{array}{l}4 \\
4 \\
5 \\
5 \\
3 \\
4 \\
5 \\
6\end{array}$ & $\begin{array}{l}4 \\
4 \\
5 \\
6 \\
3 \\
4 \\
5 \\
5\end{array}$ & $\begin{array}{l}4 \\
4 \\
5 \\
6 \\
3 \\
4 \\
5 \\
0\end{array}$ & $\begin{array}{l}4 \\
4 \\
5 \\
5 \\
4 \\
4 \\
5 \\
6\end{array}$ & $\begin{array}{l}4 \\
4 \\
5 \\
6 \\
4 \\
4 \\
5 \\
6\end{array}$ & $\begin{array}{l}3 \\
4 \\
\mathrm{I} \\
\mathrm{J} \\
3 \\
4 \\
\mathrm{~K} \\
\mathrm{~L}\end{array}$ & $\begin{array}{l}3 \\
4 \\
\mathrm{I} \\
\mathrm{J} \\
3 \\
3 \\
\mathrm{~K}\end{array}$ & $\begin{array}{l}3 \\
4 \\
\text { I } \\
\text { J } \\
4 \\
4 \\
\text { K } \\
\text { L }\end{array}$ \\
\hline $\mathbf{S}$ & $\mathbf{S}$ & $\mathbf{S}$ & S 2 & $\mathbf{Z Z}$ & $\mathbf{Z}$ & $\mathbf{Z}$ & & & & & & & & & & & & & & & & & & & & & \\
\hline 3 & 3 & 5 & 63 & 33 & 5 & 6 & 0 & 2 & 2 & 2 & 3 & 2 & 2 & 2 & 3 & 3 & 3 & 3 & 4 & 4 & 4 & 4 & 4 & 4 & 4 & 4 & 4 \\
\hline 3 & 3 & 5 & 53 & 34 & 5 & 5 & 3 & 0 & 2 & 3 & 3 & 3 & 4 & 4 & 3 & 3 & 4 & 4 & 4 & 4 & 4 & 4 & 4 & 4 & 4 & 4 & 4 \\
\hline 3 & 3 & 5 & 63 & 34 & 5 & 5 & 2 & 0 & 0 & 2 & 2 & 3 & 3 & 3 & 3 & 2 & 3 & 3 & 3 & 4 & 3 & 4 & 4 & 4 & 4 & 4 & 4 \\
\hline 3 & 3 & 5 & 63 & 34 & 5 & 6 & 0 & 0 & 0 & 0 & 0 & 2 & 2 & 2 & 2 & 2 & 2 & 2 & 2 & 3 & 3 & 3 & 3 & 3 & 4 & 4 & 4 \\
\hline 3 & 3 & 5 & 6 & 44 & 5 & 6 & 3 & 2 & 2 & 2 & 0 & 4 & 4 & 4 & 3 & 3 & 3 & 3 & 2 & 4 & 4 & 4 & 3 & 3 & 4 & 4 & 4 \\
\hline 3 & 4 & 5 & 53 & 33 & 5 & 6 & 2 & 2 & 3 & 3 & 4 & 0 & 2 & 3 & 2 & 3 & 3 & 4 & 4 & 2 & 3 & 3 & 3 & 4 & 4 & 4 & 4 \\
\hline 3 & 4 & 5 & 63 & 33 & 5 & 6 & 0 & 2 & 2 & 2 & 3 & 0 & 0 & 0 & 2 & 2 & 2 & 2 & 3 & 2 & 2 & 2 & 3 & 3 & 4 & 4 & 4 \\
\hline 3 & 4 & 6 & 63 & 33 & 5 & 6 & 2 & 3 & 3 & 3 & 4 & 3 & 2 & 0 & 4 & 3 & 3 & 2 & 4 & 4 & 3 & 3 & 4 & 4 & 4 & 4 & 4 \\
\hline 3 & 4 & 5 & 53 & 34 & 5 & 6 & 2 & 0 & 2 & 2 & 2 & 0 & 2 & 3 & 0 & 2 & 2 & 3 & 2 & 0 & 2 & 2 & 0 & 2 & 4 & 4 & 4 \\
\hline 3 & 4 & 5 & 63 & 34 & 5 & 5 & 2 & 0 & 0 & 2 & 2 & 2 & 2 & 2 & 2 & 0 & 2 & 2 & 2 & 2 & 0 & 2 & 2 & 2 & 4 & 4 & 4 \\
\hline 3 & 4 & 5 & 63 & 34 & 5 & 6 & 0 & 0 & 0 & 0 & 0 & 0 & 0 & 0 & 0 & 0 & 0 & 0 & 0 & 0 & 0 & 0 & 0 & 0 & 4 & 4 & 4 \\
\hline 3 & 4 & 6 & 63 & $\begin{array}{ll}34 \\
\end{array}$ & 45 & 6 & 2 & 3 & 2 & 2 & 2 & 3 & 2 & 0 & 3 & 2 & 2 & 0 & 2 & 3 & 2 & 2 & 3 & 2 & 4 & 4 & 4 \\
\hline 3 & 4 & 5 & 6 & 44 & 5 & 6 & 3 & 2 & 2 & 2 & 0 & 3 & 3 & 3 & 2 & 2 & 2 & 2 & 0 & 2 & 2 & 2 & 0 & 0 & 4 & 4 & 4 \\
\hline 4 & 4 & 5 & 53 & 34 & 5 & 6 & 4 & 3 & 4 & 4 & 4 & 2 & 3 & 4 & 2 & 3 & 3 & 4 & 3 & 0 & 2 & 2 & 0 & 2 & 4 & 4 & 4 \\
\hline 4 & 4 & 5 & 6 & 34 & 5 & 5 & 4 & 3 & 3 & 4 & 4 & 3 & 3 & 3 & 3 & 2 & 3 & 3 & 3 & 2 & 0 & 2 & 2 & 2 & 4 & 4 & 4 \\
\hline 4 & 4 & 5 & 63 & 34 & 5 & 6 & 3 & 3 & 3 & 3 & 3 & 2 & 2 & 2 & 2 & 2 & 2 & 2 & 2 & 0 & 0 & 0 & 0 & 0 & 4 & 4 & 4 \\
\hline 4 & 4 & 5 & 5 & 44 & 5 & 6 & 4 & 4 & 4 & 4 & 4 & 4 & 4 & 4 & 3 & 4 & 4 & 4 & 3 & 2 & 3 & 3 & 0 & 2 & 4 & 4 & 4 \\
\hline 4 & 4 & 5 & 6 & 44 & 45 & 6 & 4 & 4 & 4 & 4 & 3 & 4 & 4 & 4 & 3 & 3 & 3 & 3 & 2 & 2 & 2 & 2 & 0 & 0 & 4 & 4 & 4 \\
\hline
\end{tabular}

because of the very high incidence of incompatible matings involving plants of different class; that is, as expected, only one $S-Z$ pair in the pollen of tetraploids needs to be matched by the same pair in the stigma for incompatibility to occur (hypothesis 1 ; table 2). However, as in the case of the analysis of diploid families, a number of the pollinations shown in table 4 have been misclassified. In the great majority of such cases, misclassification has involved adjacent classes of pollination. Thus of the 463 pollinations shown in table 4,377 ( 81 per cent) were correctly classified and 86 (19 per cent) were misclassified. Of the latter, no less than 52 (60 per cent of all misclassified pollinations) concern the misclassification of incompatible pollinations as $\frac{1}{6}$ compatibles and vice versa; and a further 32 (37 per cent of the misclassifications) concern the misclassification of $\frac{1}{6}$ compatible pollinations as those scored as 2 and vice versa. Only two of these misclassifications involved shifts beyond the immediately adjacent pollination class. Thus while the proportion of misclassified tetraploid pollinations is rather higher than that obtained with diploids, it has overwhelmingly occurred where it might have been expected.

\section{(ii) Seed-set}

The average seed-set that was obtained for each of the seven categories of cross and for the 28 plants that were selfed are shown in table 4 . The 
8

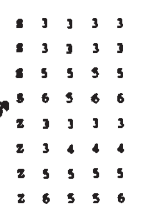

$\begin{array}{lll}3 & 3 & 3 \\ 3 & 4 & 4 \\ 5 & 5 & 5 \\ 6 & 5 & 6 \\ 4 & 3 & 3 \\ 4 & 3 & 3 \\ 5 & 5 & 5 \\ 6 & 6 & 6\end{array}$
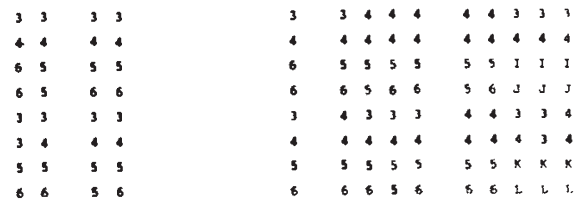

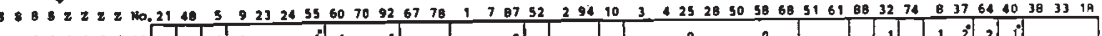
335633556

33434554

33563455

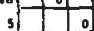

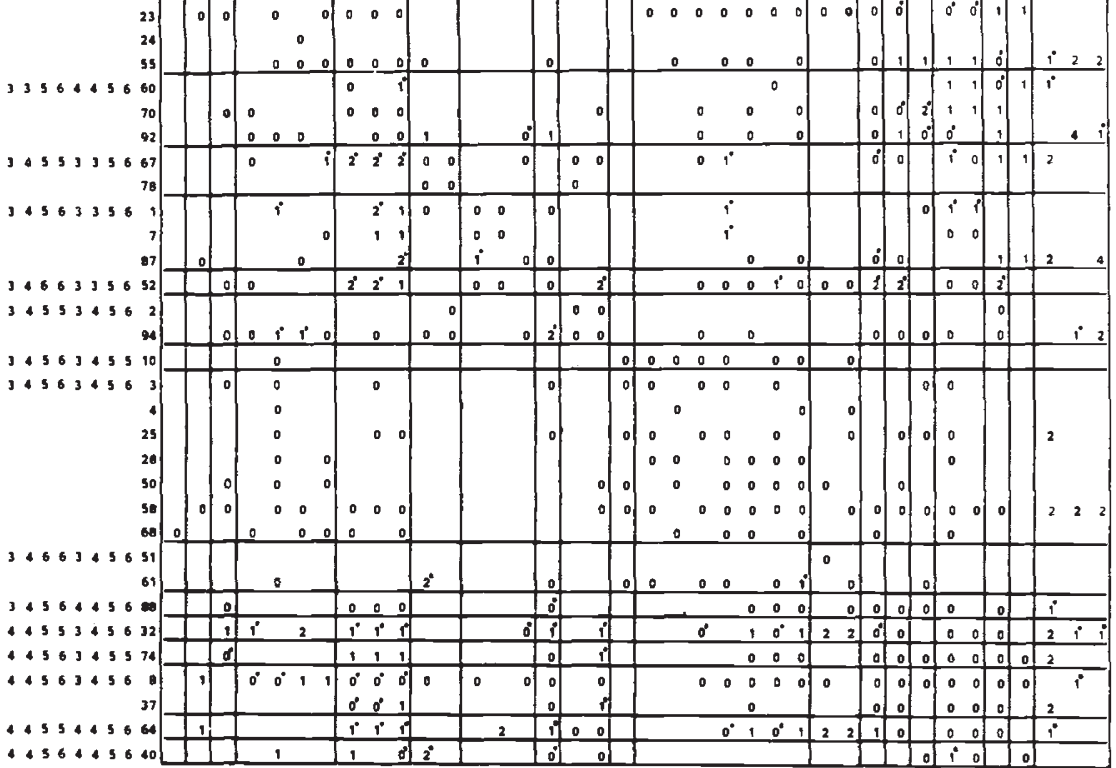

FIG. 1. The pattern of pollinations obtained when the tetraploids of families $26^{4} \times 1^{4}$ and $1^{4} \times 26^{4}$ are crossed. Pollinations were classified on the scoring system shown in table 1 . A superscript dot indicates that the classification of the cross in question is inconsistent with other crosses of the same type; see text for further details.

TABLE 4

Average percentage seed-set of selfs and crosses

$\left.\begin{array}{ccc}\hline \begin{array}{c}\text { Compatibility } \\ \text { of cross }\end{array} & \text { No. of crosses } & \begin{array}{c}\text { Average } \\ \text { seed-set }(\%)\end{array} \\ \hline \text { Selfs } & 28 & 0.14 \\ 0 & 50 & 0.77 \\ \frac{1}{6} & 9 & 3.16 \\ \frac{11}{36} & 11 & 2.70 \\ \frac{1}{2} & 3 & 1.22 \\ \frac{7}{12} & 9 & 3.33 \\ \frac{3}{4} & 6 & 2.94 \\ 1 & 8 & 1.58\end{array}\right] 2.65$


very low seed-set obtained from the selfs $(0 \cdot 14$ per cent) shows that tetraploid $L$. perenne is highly self-incompatible. The average seed-set of the 50 incompatible crosses, though still low, is nevertheless significantly greater than that of the selfs (table 5(a)). We have previously found such a difference with $L$. multiflorum (Fearon et al., 1983), though the cause in neither case is clear.

\section{TABLE 5}

Analysis of variance of the seed-set data from (a) selfs and incompatible crosses; (b) compatible crosses; and (c) incompatible and compatible crosses; the data were transformed from percentages to angles prior to analysis. The binomial error is $820 \cdot 7 / N$, where $N$ is the harmonic mean of the estimated number of florets per unit

\begin{tabular}{|c|c|c|}
\hline Item & d.f. & M.S. \\
\hline \multicolumn{3}{|l|}{ (a) } \\
\hline Selfs v. Crosses & 1 & $64 \cdot 408^{* *}$ \\
\hline Within selfs and crosses & 76 & $7 \cdot 486 * * *$ \\
\hline Binomial error & $\infty$ & 1.655 \\
\hline \multicolumn{3}{|l|}{ (b) } \\
\hline Between compatible categories & 5 & $15 \cdot 098$ \\
\hline Within categories & 40 & $39 \cdot 505^{* * *}$ \\
\hline Binomial error & $\infty$ & $1 \cdot 748$ \\
\hline \multicolumn{3}{|l|}{ (c) } \\
\hline Incompatible crosses v. compatible crosses & 1 & $280 \cdot 013^{* * *}$ \\
\hline Within incompatible and compatible crosses & 94 & $23 \cdot 297^{* * *}$ \\
\hline Binomial error & $\infty$ & $1 \cdot 648$ \\
\hline
\end{tabular}

The analysis of variance of the seed-set of the six categories of compatible cross (table 5(b)) shows that there is no evidence in these data of any effect of the level of compatibility on seed-set. (The very low overall average seed-set of these crosses is due to the unfavourable conditions that obtained during the course of this experiment.) The most important comparison that can be made with these data is that between the seed-set of incompatible and compatible crosses which is highly significant (table $5(\mathrm{c})$ ). Thus while these data cannot be used to confirm the classification of the plants of these tetraploid families in respect of compatible crosses, there is little doubt that this classification has at least separated incompatible from compatible crosses. Lastly, we note that for all three comparisons, the within crosses and/or selfs item is significantly greater than the binomial error which indicates that, in general, the variation within categories is much greater than would be expected from random causes alone.

\section{Discussion}

In deriving the expectations shown in table 1 , it has been assumed that the alleles at each locus act independently in the pollen; that is, there is no dominance. We are now in a position to test this assumption. Consider, for example, the cross $S_{4456} Z_{3456} \times S_{3456} Z_{3456}$ which in the absence of dominance in the pollen is expected to be incompatible on hypothesis 1 . However, 


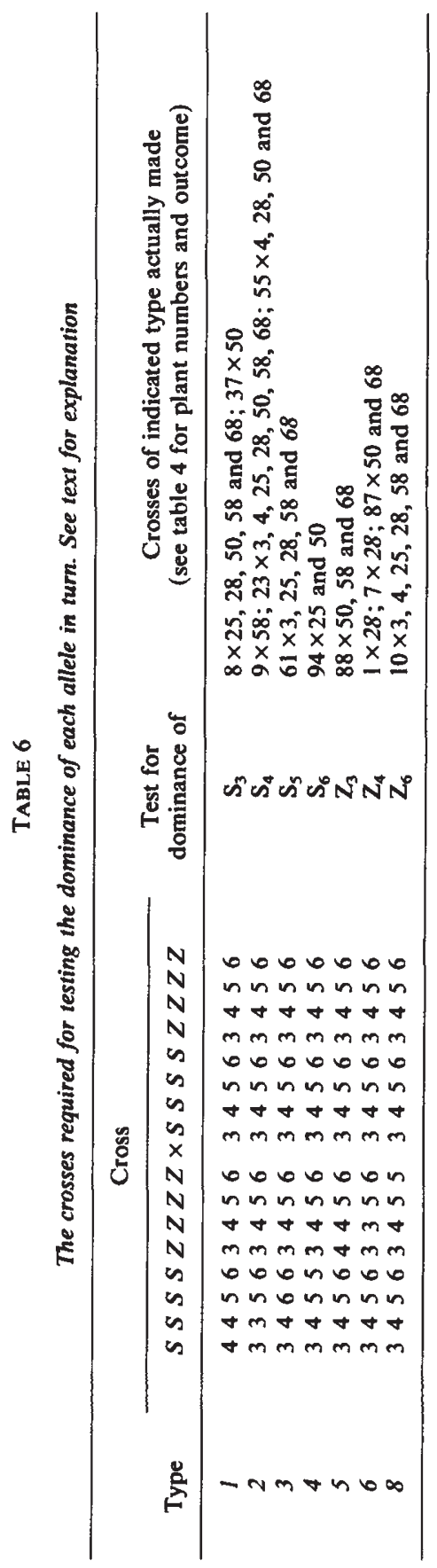


if $S_{3}$ is dominant to any one of the other three $S$ alleles, the cross is expected to be $\frac{1}{6}$ compatible, since $\frac{1}{6}$ of the pollen will be $S_{3(4)} Z_{--}, S_{3(5)} Z_{-- \text {, or }} S_{3(6)} Z_{-}$, $S_{3(6)} Z_{-}$, as the case may be. Similarly, the cross is expected to be $\frac{1}{3}$ compatible if $S_{3}$ is dominant to two of the remaining $S$ alleles; and $\frac{1}{2}$ compatible if it is dominant to all three. Each of the $S$ and $Z$ alleles can be similarly tested against the others by examining the compatibility of crosses between females that possess all of the alleles at each locus except the one under test and males which are doubly tetra-allelic, for all such crosses are expected to be incompatible on hypothesis 1 in the absence of dominance. However, because the genotype $S_{3456} Z_{3466}$ does not occur in the present experiment, it is not possible to test for the dominance of the $Z_{5}$ allele. The seven types of cross that can be examined are shown in table 6. Thirty-five of the 38 appropriate crosses have been scored as incompatible and three as $\frac{1}{6}$ compatible (fig. 1). Since the latter are distributed over two different types of cross (types 3 and 6), other examples of which, in both cases, were scored as incompatible, it is almost certain that these exceptional pollinations are due to misclassification. Taken as a whole, therefore, these data leave little doubt that the alleles at both loci act independently in the pollen, as well as the stigmas, of tetraploids.

The observation that all of these crosses are incompatible, also rules out the possibility that the pattern of pollinations obtained in these tetraploid families (fig. 1) could be explained on hypothesis 2 with dominance of the alleles in the pollen. Thus, consider again the cross $S_{4456} Z_{3456} \times S_{3456} Z_{3456}$, which in the absence of dominance is expected to be half-compatible on this hypothesis. However, if $S_{3}$ were to be recessive to $S_{4}, S_{5}$ and $S_{6}$, this cross would be incompatible; that is, this cross provides a test for the recessivity of $S_{3}$, the allele missing from the female parent. The other crosses listed in table 6 provide similar tests of recessivity for remaining alleles. The fact that nearly all of these crosses were scored as incompatible implies, on this argument, that each of the alleles is recessive in turn to the others, which is clearly impossible. Hence, the possibility that the incompatibility of these crosses is due to dominance on hypothesis 2 can be rejected.

There is one further test that can be applied to the data shown in fig. 1. Hitherto it has been supposed that incompatibility occurs when an $S-Z$ pair in the pollen is matched by the same pair in the stigma. Though unlikely, it is possible that the alleles need be matched at one locus alone for incompatibility to occur. This possibility, however, can be dismissed for the following reasons. If matching of alleles at the $Z$ locus alone is sufficient for incompatibility, then the cross $S_{3356} Z_{3456} \times S_{4455} Z_{3456}$ should be incompatible. Two crosses of this type occur in the data, involving plant no. 32 used as female and plant nos. 9 and 24 used as males; in each case, these crosses were scored as partially compatible. If, on the other hand, matching of the alleles at the $S$ locus alone were sufficient for incompatibility then the cross $S_{3456} Z_{3356} \times S_{3356} Z_{4456}$ should be incompatible; again all such crosses, which involved plant nos. 1,7 and 87 used as females and plant nos. 70 and 92 as males, were scored as partially compatible. The possibility that matching of alleles at one locus alone is sufficient for incompatibility can therefore be rejected.

These results confirm in all respects the classification and conclusions of the first two stages of the three part procedure used to elucidate the inheritance and expression of self-incompatibility in tetraploid ryegrass 
(Fearon et al., 1984a, b). The chief conclusions are that the alleles at both loci act independently both in the pollen and the stigma, and that only one $S-Z$ pair in the pollen needs to be matched by the same pair in the stigma for the pollen to be incompatible. The results also show that there is no evidence of dosage effects in the stigma and that the chromosomes assort at random at meiosis with respect to these loci. Lastly, that only 18 of the expected 81 different incompatibility classes have turned up in these families is simply due to the relatively small number of plants that have been examined in this experiment. The number required to stand a reasonable chance of obtaining at least one representative of each of the expected 81 classes is, of course, impracticably large.

That only one $S-Z$ pair in the pollen needs to be matched for incompatibility to occur, despite the presence of as many as three other compatible $S-Z$ combinations strongly suggests that the growth of incompatible pollen is positively inhibited, rather than that of compatible pollen being positively stimulated.

Lastly, we note that the level of cross-compatibility in a full-sib family of tetraploids is much less than in an equivalent family of diploids. This observation raises the question of the level of cross-compatibility in a population of tetraploids compared with that in one of diploids in which the same number of $S$ and $Z$ alleles are segregating. Lundqvist (1963) gave graphs showing the relationship between cross-compatibility and number of alleles in populations of diploids and tetraploids for species with a two-locus, multi-allelic system of self-incompatibility, though no details were given of the equations from which these estimates were obtained. We have, therefore, re-derived these equations, details of which are given in Scarrott (1981). Like Lundqvist, we have assumed that the same number of alleles occurs at both the $S$ and $Z$ loci; that these alleles are equally frequent; that the genotype frequencies in the population are in deterministic equilibrium; and that the chromosomes assort at random at meiosis in the tetraploids with respect to $S$ and $Z$. The results of these calculations, which confirm those of Lundqvist (loc. cit.), are shown in table 7. It is clear from this table that whereas as few as four alleles will give over 95 per cent cross-compatibility in a population of diploids, eight are needed for the same level in a population of tetraploids. However, as soon as the number of alleles at each locus is twelve or more, this difference, for all practical purposes, vanishes. Since the alleles at each locus are subject to frequency dependent selection, it is, perhaps, unlikely that their number would be low enough in a natural population of tetraploids to have much effect on the amount of seed set, unless, that is, the population had been founded in isolation from a very small number of plants or had gone through a bottleneck. On the other hand, in deriving the estimates shown in table 7, it has been assumed that the number and frequency of the alleles at each locus is the same; if this is not true, the percentage cross-compatibility in the population would be lower than indicated in the table. However, since virtually nothing is known about the properties of this polymorphism in natural populations, the validity of these assumptions must remain for the present a matter for conjecture.

These calculations have, however, more immediate implications for the forage plant breeder, for they indicate quite clearly the risk of founding a new variety of a tetraploid grass on a very small number of basic plants. 


\section{TABLE ?}

The percentage of compatible crosses in a diploid and an autotetraploid population of a self-incompatible species of grass assuming that the same number, $n$, of alleles occurs at each locus, that these alleles are equally frequent and that the population is in equilibrium

\begin{tabular}{rcc}
\hline & \multicolumn{2}{c}{ Compatible crosses (\%) } \\
\cline { 2 - 3 } & Diploid & Tetraploid \\
\hline 2 & 55.56 & 12.43 \\
3 & 90.28 & 40.22 \\
4 & 96.67 & 61.64 \\
5 & 98.56 & 77.66 \\
6 & 99.27 & 87.33 \\
7 & 99.60 & 92.73 \\
8 & 99.76 & 95.72 \\
9 & 99.85 & 97.40 \\
10 & 99.90 & 98.37 \\
11 & 99.93 & 98.95 \\
12 & 99.95 & 99.30 \\
13 & 99.96 & 99.53 \\
14 & 99.97 & 99.67 \\
15 & 99.98 & 97.77 \\
20 & 99.99 & 99.95 \\
\hline
\end{tabular}

Bearing in mind that a newly-induced autotetraploid will possess, at best, only two different alleles at each locus, a cross between a pair of such plants would yield a progeny in which only four different alleles would be segregating at each locus, even if these parents were quite unrelated. A variety founded on only two plants, therefore could be expected to have only a 61.64 per cent cross-compatibility, which might well be less than is desirable for seed-multiplication. It is preferable, therefore, not to found varieties on a very small number of basic plants and to ensure that the founder plants used carry as few alleles in common as possible at either locus.

Acknowledgements. We wish to acknowledge the award of a Science and Engineering Research Council CASE studentship to C.H.F. and an A.R.C. research grant to M.J.L. which enabled this work to be carried out.

\section{REFERENCES}

CORNISH, M. A., HAYWARD, M. D. AND LAWRENCE, M. J. 1980. Self-incompatibility in ryegrass. IV. Seed-set in diploid Lolium perenne L. Heredity, 44, 333-340.

FEARON, C. H., HAYWARD, M. D. AND LAWRENCE, M. J. 1983. Self-incompatibility in ryegrass. V. Genetic control, linkage and seed-set in diploid Lolium multiflorum Lam. Heredity, 50, $35-45$.

FEARON, C. H., HAYWARD, M. D. AND LAWRENCE, M. J. 1984a. Self-incompatibility in ryegrass. VII. The determination of incompatibility genotypes in autotetraploid families of Lolium perenne L. Heredity 53, 403-413.

FEARON, C. H., HAYWARD, M. A. AND LAWRENCE, M. J. 1984b. Self-incompatibility in ryegrass. VIII. The mode of action of $S$ and $Z$ alleles in the pollen of autotetraploids of Lolium perenne L. Heredity 53 415-422. 
LUNDQVIST, A. 1963. Self-incompatibility and the breeding of herbage grasses. In Recent Plant Breeding Research, 1946-1961. E. Aakerberg, A. Hagberg, G. Olsson and O. Tedin (ed.), 192-202.

SCARROTT, C. H. 1981. Self-incompatibility in Diploid and Tetraploid Lolium Species. Ph.D. thesis, University of Birmingham. 